\title{
ON QUANTUM SPACES OF LIE ALGEBRAS
}

\author{
LIEVEN LE BRUYN AND MICHEL VAN DEN BERGH
}

(Communicated by Maurice Auslander)

\begin{abstract}
The homogenization $H(\mathfrak{g})$ of the enveloping algebra of a finite dimensional Lie algebra $\mathfrak{g}$ is an Artin-Schelter regular algebra. We characterize $d$-dimensional linear subspaces in the corresponding quantum space $\mathbb{P}_{q}(\mathfrak{g})$ as homogenizations of induced representations from codimension $d$ Lie subalgebras. Furthermore we prove that the point variety has an embedded component iff there is a line, not contained in this point variety.
\end{abstract}

\section{INTRODUCTION AND NOTATION}

To any finite-dimensional Lie algebra

$$
\mathfrak{g}=\mathbb{C} X_{1} \oplus \cdots \oplus \mathbb{C} X_{n} \quad \text { with }\left[X_{i}, X_{j}\right]=\sum_{k=1}^{n} \alpha_{i j, k} X_{k}
$$

one classically associates an associative algebra $U(\mathfrak{g})$, the so-called "enveloping algebra" of $\mathfrak{g}$. The enveloping algebra carries a natural filtration

$$
U_{0}(\mathfrak{g}) \subset U_{1}(\mathfrak{g}) \subset \cdots \subset U_{n}(\mathfrak{g}) \subset \cdots
$$

such that $\operatorname{gr} U(\mathfrak{g})=S(\mathfrak{g})$, the symmetric algebra on $\mathfrak{g}$. This is the famous Poincaré-Birkhoff-Witt theorem.

By homogenizing the defining relations of $U(\mathfrak{g})$ one can also associate a regular algebra in the sense of Artin and Schelter [1] to $\mathfrak{g}$. This algebra, which will be denoted by $H(\mathfrak{g})$ in the sequel, is the positively graded quadratic algebra generated by $X_{0}, \ldots, X_{n}$ where $X_{0}$ is taken to be central and the remaining defining relations are

$$
X_{i} X_{j}-X_{j} X_{i}-\sum_{i=1}^{n} \alpha_{i j, k} X_{k} X_{0}
$$

Observe that $H(\mathfrak{g}) /\left(X_{0}-1\right) H(\mathfrak{g}) \cong U(\mathfrak{g})$, the enveloping algebra of $\mathfrak{g}$, and that $\left.H(\mathfrak{g}) / X_{0} H(\mathfrak{g}) \cong \mathrm{gr} U(\mathfrak{g})\right) \cong S(\mathfrak{g})$. From these facts one derives as usual (see, e.g., [8] or [9]) that $H(\mathfrak{g})$ is a Noetherian maximal order domain, that it is Auslander-regular of dimension $n+1$, and that it satisfies the Cohen-Macaulay property.

Received by the editors October 18, 1991 and, in revised form, February 26, 1992.

1991 Mathematics Subject Classification. Primary 16S30.

The first author is a research associate of the NFWO (Belgium). 
Following Artin [3] we define the quantum space $\mathbb{P}_{q}(\mathfrak{g})$ of $\mathfrak{g}$ to be $\operatorname{Proj}(H(\mathfrak{g}))$, which is the quotient category of the category of all finitely generated graded left $H(\mathfrak{g})$-modules modulo the subcategory of finite length modules. Via the identification $S(\mathfrak{g})=H(\mathfrak{g}) / X_{0} H(\mathfrak{g}), \mathbb{P}\left(\mathfrak{g}^{*}\right)=\operatorname{Proj} S(\mathfrak{g})$ will be thought of as the hyperplane at infinity of $\mathbb{P}_{q}(\mathfrak{g})$ with equation $X_{0}=0$.

As in [2] one can define points and lines in $\mathbb{P}_{1}(\mathfrak{g})$. This note originated from our attempts to understand the following example, taken from [7].

Example 1.1. Let $\mathfrak{s l}(2, \mathbb{C})=\mathbb{C} e \oplus \mathbb{C} f \oplus \mathbb{C} h$ with $[e, f]=h,[h, e]=2 e$, and $[h, f]=-2 f$. Then the lines in $\mathbb{P}_{q}(\mathfrak{s l}(2, \mathbb{C}))$ are precisely the lines lying on the pencil of quadrics in $\mathbb{P}^{3}=\operatorname{Proj}(\mathbb{C}[e, f, h, t])$

$$
Q(\delta)=\mathscr{V}\left(\left(h^{2}+4 e f\right)+\delta^{2} t^{2}\right) .
$$

In particular, all lines intersect the hyperplane $V(t)$ at infinity in a point of the conic $\left(t, h^{2}+4 e f\right)$, which is precisely the embedded component of the point-variety of $\mathbb{P}_{q}(\mathfrak{s l}(2, \mathbb{C}))$.

Moreover, all three-dimensional Lie algebras behave in a similar way. That is, the point-variety has an embedded component lying in the plane at infinity and all lines not lying on the point-variety intersect with the hyperplane at infinity in a point of this component.

In this note we partially generalize Example 1.1 by showing that there always is a subvariety of the hyperplane at infinity in $\mathbb{P}_{q}(\mathfrak{g})$, closely related with a possible embedded component of the point variety, such that all lines, not lying in the hyperplane at infinity, intersect this variety. In case $[\mathfrak{g}, \mathfrak{g}]=\mathfrak{g}$ this variety is the embedded component of the point variety.

We also show that the existence of such an embedded component is caused by the fact that there may be lines in $\mathbb{P}_{q}(\mathfrak{g})$, not lying in the point variety of $\mathbb{P}_{q}(\mathfrak{g})$.

The proofs of these results, given in $\S 3$, rely on the classification of linear subspaces in $\mathbb{P}_{q}(\mathfrak{g})$, given in the next section.

\section{LINEAR SUBSPACES, LIE SUBALGEBRAS, AND POLARIZATIONS}

We first state a few generalities. To any finitely generated filtered $U(\mathfrak{g})$ module

$$
\cdots \subset M_{-1} \subset M_{0} \subset M_{1} \subset \cdots \subset \bigcup_{i=-\infty}^{\infty} M_{i}=M,
$$

we can associate a graded $H(\mathfrak{g})$-module (and hence an object in $\left.\mathbb{P}_{q}(\mathfrak{g})\right)$ :

$$
\widetilde{M}=\bigoplus_{i=-\infty}^{\infty} M_{i} X_{0}^{i} \subset M\left[X_{0}, X_{0}^{-1}\right] .
$$

We observe that $\widetilde{M} /\left(X_{0}-1\right) \widetilde{M} \cong M$ and conversely, any $X_{0}$-torsionfree graded left $H(\mathfrak{g})$-module arises in this way, see [4] or [6].

In the sequel there will be a few occasions where we will use the notation $\widetilde{M}$ for a graded $H(\mathfrak{g})$-module, even if it is not a priori obtained from some filtered $U(\mathfrak{g})$-module $M$; i.e., $\widetilde{M}$ could have $X_{0}$-torsion.

In this note we concentrate on a specific class of graded left $H(\mathfrak{g})$-modules. 
Definition 2.1. A $d$-dimensional linear subspace of $\mathbb{P}_{q}(\mathfrak{g})$ is the object in $\mathbb{P}_{q}(\mathfrak{g})$ associated to a cyclic graded left $H(\mathfrak{g})$-module $\widetilde{M}=\bigoplus_{i=0}^{\infty} M_{i}$ with Hilbert series $\mathscr{H}(\widetilde{M}, t)=\sum_{i=0}^{\infty} \operatorname{dim}_{\mathbb{C}}\left(M_{i}\right) t^{i}=1 /(1-t)^{d+1}$.

Theorem 2.2. The d-dimensional linear subspaces of $\mathbb{P}_{q}(\mathfrak{g})$ are:

(1) The d-dimensional subspaces in $\mathbb{P}^{n-1}=\operatorname{Proj}(S(\mathfrak{g}))$,

(2) The homogenizations $\widetilde{M}$ of induced modules

$$
M=U(\mathfrak{g}) \otimes_{U(\mathfrak{h})} \mathbb{C}_{f}
$$

where $\mathfrak{h}$ is a Lie subalgebra of $\mathfrak{g}$ of codimension d and $\mathbb{C}_{f}$ is the one-dimensional representation of $\mathfrak{h}$ determined by $f \in \mathfrak{h}^{*}$ such that $f([\mathfrak{h}, \mathfrak{h}])=0$. Here the pair $(\mathfrak{h}, f)$ is uniquely determined by $\widetilde{M}$.

Proof. Let $\widetilde{M}$ be a graded $H(\mathfrak{g})$-module as in Definition 2.1. Then it is easy to see that $\widetilde{M}$ is either annihilated by $X_{0}$ or $X_{0}$ acts without torsion. In the first case $\widetilde{M}$ will be a quotient of $H(\mathfrak{g}) / X_{0} H(\mathfrak{g})=S(\mathfrak{g})$; i.e., $\widetilde{M}$ will represent a linear subspace in Proj $S(\mathfrak{g})$.

In the second case $M=M /\left(X_{0}-1\right) \widetilde{M}$ is a filtered $\left(H(\mathfrak{g}) /\left(X_{0}-1\right) H(\mathfrak{g})=\right.$ $U(\mathfrak{g}))$-module, i.e., there is a map $\phi: U(\mathfrak{g}) \rightarrow M$ such that

$$
\operatorname{dim} \phi\left(U_{i}(\mathfrak{g})\right)=\left(\begin{array}{c}
d+i \\
i
\end{array}\right) .
$$

The result now follows from the following proposition.

Proposition 2.3. Assume that $M$ is a left $U(\mathfrak{g})$-module and that there is a surjective map $\phi: U(\mathfrak{g}) \rightarrow M$ such that

$$
\operatorname{dim} \phi\left(U_{i}(\mathfrak{g})\right)=\left(\begin{array}{c}
d+i \\
i
\end{array}\right) \quad \text { for } i \in \mathbb{N} .
$$

Then there is a unique pair $(\mathfrak{h}, f)$ such that

$$
M \cong U(\mathfrak{g}) \otimes_{U(\mathfrak{h})} \mathbb{C}_{f}
$$

with notation as in Theorem 2.2 .

Furthermore the filtration induced on $M$ via $\phi$ makes (2) into a filtered isomorphism.

Proof. The uniqueness of the pair $(\mathfrak{h}, f)$ is easy to see. Therefore we concentrate on its existence. First define

$$
\mathfrak{h}^{\prime}=U_{1}(\mathfrak{g}) \cap \operatorname{ker} \phi .
$$

Clearly $\operatorname{dim} \mathfrak{h}^{\prime}=n-d$.

Using the fact that $U(\mathfrak{g})$ has a PBW-basis, it is easy to see that

$$
U_{1}(\mathfrak{g}) \mathfrak{h}^{\prime} \cap U_{1}(\mathfrak{g})=\left[\mathfrak{h}^{\prime}, \mathfrak{h}^{\prime}\right]+\mathfrak{h}^{\prime} .
$$

Furthermore if $V$ is the image of $U_{1}(\mathfrak{g}) h^{\prime}$ in $U_{2}(\mathfrak{g}) / U_{1}(\mathfrak{g})=S_{2}(\mathfrak{g})$ then we compute that

$$
\operatorname{dim} V=\left(\begin{array}{c}
n+1 \\
2
\end{array}\right)-\left(\begin{array}{c}
d+1 \\
2
\end{array}\right) .
$$


Hence we obtain

$$
\begin{aligned}
\operatorname{dim} U_{1}(\mathfrak{g}) \mathfrak{h}^{\prime} & =\operatorname{dim} V+\operatorname{dim} U_{1}(\mathfrak{g}) \mathfrak{h}^{\prime} \cap U_{1}(\mathfrak{g}) \\
& =\left(\begin{array}{c}
n+1 \\
2
\end{array}\right)-\left(\begin{array}{c}
d+1 \\
2
\end{array}\right)+\operatorname{dim}\left(\left[\mathfrak{h}^{\prime}, \mathfrak{h}^{\prime}\right]+\mathfrak{h}^{\prime}\right)
\end{aligned}
$$

Also, using (1) and the fact that $U_{1}(\mathfrak{g}) \subset \operatorname{ker} \phi$ we find that

$$
U_{1}(\mathfrak{g}) \mathfrak{h}^{\prime}+\left(\begin{array}{c}
d+2 \\
2
\end{array}\right) \leq\left(\begin{array}{c}
n+2 \\
2
\end{array}\right)
$$

Combining (3) and (4) we find

$$
\operatorname{dim}\left(\left[\mathfrak{h}^{\prime}, \mathfrak{h}^{\prime}\right]+\mathfrak{h}^{\prime}\right) \leq n-d=\operatorname{dim} \mathfrak{h}^{\prime}
$$

Hence $\left[\mathfrak{h}^{\prime}, \mathfrak{h}^{\prime}\right] \subset \mathfrak{h}^{\prime}$ and thus we find that $\mathfrak{h}^{\prime}$ is a Lie subalgebra of $U_{1}(\mathfrak{g})$.

Let $\mathfrak{h}$ be the image of $\mathfrak{h}^{\prime}$ in $\mathfrak{g}$ under the canonical decomposition $U_{1}(\mathfrak{g})=$ $\mathbb{C} \oplus \mathfrak{g}$. Since $1 \notin \mathfrak{h}^{\prime}$, there will be a linear map $f \in \mathfrak{h}^{*}$ such that

$$
\mathfrak{h}^{\prime}=\{u-f(u) \mid u \in \mathfrak{h}\} \text {. }
$$

Let $u, v \in \mathfrak{h}$. Then

$$
[u-f(u), v-f(v)]=[u, v] \in \mathfrak{h}^{\prime}
$$

and therefore $f([u, v])=0$.

We now have a surjective map

$$
U(\mathfrak{g}) / U(\mathfrak{g}) \mathfrak{h}^{\prime} \cong U(\mathfrak{g}) \otimes_{U(\mathfrak{h})} \mathbb{C}_{f} \rightarrow M
$$

Applying (1) yields that this map must be a filtered isomorphism.

To any $d$-dimensional linear subspace in $\mathbb{P}_{q}(\mathfrak{g})$ we can associate a unique $d$-dimensional linear subspace in $\mathbb{P}^{n}=\mathbb{P}\left(\mathfrak{g}^{*} \oplus \mathbb{C} X_{0}^{*}\right)$ as follows. Let $\widetilde{M}$ be a graded quotient $H(\mathfrak{g}) \rightarrow \widetilde{M}$. Then restriction to degree 1 defines a linear map $\mathfrak{g} \oplus \mathbb{C} X_{0} \rightarrow \widetilde{M}_{1}$, hence a linear subspace in $\mathbb{P}^{n}$. Conversely, a linear subspace in $\mathbb{P}^{n}$ can at most correspond to one linear subspace in Proj $H(\mathfrak{g})$.

If we identify the open affine piece $X_{0}=1$ with $\mathfrak{g}^{*}$ we would like to know the minimal dimension $d(f)$ of a linear subspace in $\mathbb{P}_{q}(\mathfrak{g})$ containing $f \in \mathfrak{g}^{*}$.

If $f \in \mathfrak{g}^{*}$ then we denote by $B_{f}$ the bilinear form $(x, y) \rightarrow f([x, y])$ and with $\mathfrak{g}^{f}=\{x \in \mathfrak{g} \mid f([x, \mathfrak{g}])=0\}$ the radical, which is a Lie subalgebra of $\mathfrak{g}$, and as $B_{f}$ induces a nondegenerate alternating bilinear form on $\mathfrak{g} / \mathfrak{g}^{f}$ we have that $n-\operatorname{dim}\left(\mathfrak{g}^{f}\right)$ is even.

In view of Theorem 2.2 we see that a $d$-dimensional linear subspace of $\mathbb{P}_{q}(\mathfrak{g})$ containing $f$ is the homogenization of an induced one-dimensional representation of a codimension $d$ Lie subalgebra $\mathfrak{h}$ such that $f([\mathfrak{h}, \mathfrak{h}])=0$. In the terminology of $[5$, Chapter $I, \S 12] \mathfrak{h}$ is subordinate to $f$. One knows that the maximal dimension of such a Lie subalgebra is $\frac{1}{2}\left(n+\operatorname{dim}\left(\mathfrak{g}^{f}\right)\right)$, meaning that

$$
d(f) \geq \frac{1}{2}\left(n-\operatorname{dim}\left(\mathfrak{g}^{f}\right)\right),
$$

and if equality occurs then $\mathfrak{h}$ is said to be a polarization of $\mathfrak{g}$ at $f$. Note that polarizations exist for every $f$ if $\mathfrak{g}$ is solvable or $\mathfrak{g}=\mathfrak{s l}(n)$ and for arbitrary $\mathfrak{g}$ a (solvable) polarization exists whenever $f$ is a regular form (cf. [5, Chapter I, §11]). 
Corollary 2.4. The minimal dimension $d$ such that every point in $\mathbb{P}\left(\mathfrak{g}^{*} \oplus \mathbb{C} X_{0}^{*}\right)$ is contained in a d-dimensional linear subspace of $\mathbb{P}_{q}(\mathfrak{g})$ is equal to $\frac{1}{2}(n-r)$ where $r$ is the index of $\mathfrak{g}$.

If $\mathfrak{g}$ is semisimple, the index of $\mathfrak{g}$ is equal to its rank. Moreover, in this case all linear subspaces in $\mathbb{P}_{q}(\mathfrak{g})$, not lying in the hyperplane at infinity and of minimal dimension, are obtained by parabolic induction.

\section{POINTS, LINES, AND EMBEDDED COMPONENTS}

Recall from [2] that the bihomogeneous equations for the point-variety of $H(\mathfrak{g})$ are given by

$$
\begin{aligned}
x_{i}^{(1)} x_{j}^{(2)}-x_{j}^{(1)} x_{i}^{(2)} & =\left(\sum \alpha_{i j, k} x_{k}^{(1)}\right) x_{0}^{(2)}, \\
x_{0}^{(1)} x_{i}^{(2)} & =x_{i}^{(1)} x_{0}^{(2)},
\end{aligned}
$$

where $1 \leq i, j, k \leq n$.

To simplify some of the notation in the sequel, let us make the following conventions. If $U=\sum u_{i} X_{i} \in \mathfrak{g}$ then $u=\sum u_{1} x_{1}, u^{(1)}=\sum u_{i} x_{i}^{(1)}$, etc. Similarly, if $U, V \in \mathfrak{g}$ and $[U, V]=\sum w_{i} X_{i}$ then $[u, v]=\sum w_{i} x_{i}$. As above, $U$ and $V$ may be equipped with the superscripts (1) and (2).

Using this notation we may rewrite (5) more succinctly as

$$
\begin{aligned}
x_{i}^{(1)} x_{j}^{(2)}-x_{j}^{(1)} x_{i}^{(2)} & =\left[x_{i}^{(1)}, x_{j}^{(1)}\right] x_{0}^{(2)}, \\
x_{0}^{(1)} x_{i}^{(2)} & =x_{i}^{(1)} x_{0}^{(2)} .
\end{aligned}
$$

These equations determine a variety $X \subset \mathbb{P}\left(\mathfrak{g}^{*} \oplus \mathbb{C} x_{0}^{*}\right) \times \mathbb{P}\left(\mathfrak{g}^{*} \oplus \mathbb{C} x_{0}^{*}\right)$.

Let $\sigma: Y=p r_{1}(X) \rightarrow Y^{\prime}=p r_{2}(Y)$ be the induced automorphism, which is given by shifting point modules one place to the right (we are working with left modules instead of right modules as in [2]). From the description (Theorem 2.2) of points as one-dimensional representations of $U(\mathfrak{g})$ or points at infinity it follows that $\sigma$ is the identity on $Y_{\text {red }}$.

Theorem 3.1. If we set $x_{i}=x_{i}^{(1)}$, then the defining relations for $Y$ are

$$
\begin{aligned}
\left(x_{i}\left[x_{j}, x_{k}\right]+x_{j}\left[x_{k}, x_{i}\right]+x_{k}\left[x_{i}, x_{j}\right]\right) x_{0} & =0, \\
{\left[x_{i}, x_{j}\right] x_{0}^{2} } & =0 .
\end{aligned}
$$

Proof. We will cover $Y$ by affine opens. First assume that $x_{0}=x_{0}^{(1)}=1$. Then from $\sigma=$ id on $Y_{\text {red }}$ we may assume that $x_{0}^{(2)}=1$. Then from (7) we obtain that $x_{i}^{(1)}=x_{i}^{(2)}$. Substituting this in (6) yields that $\left[x_{i}, x_{j}\right]=0$ for $i, j \in\{1, \ldots, n\}$. This set of equations clearly defines the same variety as (8) when $x_{0}=1$.

Assume now that $x_{s}=x_{s}^{(1)}=1$ for some $s \in\{1, \ldots, n\}$. Again we may assume that $x_{s}^{(2)}=1$. Put $\varepsilon_{j}=x_{j}^{(1)}-x_{j}^{(2)}$. Clearly $\varepsilon_{s}=0$, and from (7) it follows that $\varepsilon_{0}=0$.

Equations (6) and (7) may now be rewritten as

$$
\begin{aligned}
x_{j} \varepsilon_{k}-x_{k} \varepsilon_{j} & =\left[x_{j}, x_{k}\right] x_{0}, \\
x_{0} \varepsilon_{j} & =0,
\end{aligned}
$$

$j, k \in\{1, \ldots, n\}$. 
Applying (9) with $j=s$ yields

$$
\varepsilon_{k}=\left[x_{s}, x_{k}\right] x_{0} .
$$

Resubstituting yields the equations

$$
\begin{array}{r}
\left(x_{s}\left[x_{j}, x_{k}\right]+x_{k}\left[x_{s}, x_{j}\right]+x_{j}\left[x_{k}, x_{s}\right]\right) x_{0}=0, \\
{\left[x_{s}, x_{j}\right] x_{0}^{2}=0,}
\end{array}
$$

where $j, k \in\{1, \ldots, n\}$. Using the fact that $x_{s}=1$, we find that the equations (11) are equivalent with the equations (8).

Now we give an interpretation for the two sets of equations occurring in (8).

Lemma 3.2. (1) $A=V\left(\left[x_{i}, x_{j}\right] ; 1 \leq i, j \leq n\right) \subset \mathbb{P}\left(\mathrm{g}^{*}\right)$ is the linear space of one-dimensional representations of $\mathfrak{g}$.

(2) $B=V\left(x_{i}\left[x_{j}, x_{k}\right]+x_{j}\left[x_{k}, x_{i}\right]+x_{k}\left[x_{i}, x_{j}\right] ; 1 \leq i, j, k \leq n\right) \subset \mathbb{P}\left(\mathfrak{g}^{*}\right)$ classifies codimension one Lie subalgebras of $\mathfrak{g}$.

Proof. (1) is clear. A point of $B$ is a surjective map $\phi: \mathfrak{g} \rightarrow \mathbb{C}$ satisfying $\phi(u) \phi([v, w])+\phi(v) \phi([w, u])+\phi(w) \phi([u, v])=0$. Let $\mathfrak{h}=\operatorname{ker}(\phi)$ and take $u, v \in \mathfrak{h}$ and $w \notin \mathfrak{h}$. It follows that $\phi([u, v])=0$; i.e., $\mathfrak{h}$ is a codimension one Lie subalgebra. Conversely the quotient map $\mathfrak{g} \rightarrow \mathfrak{g} / \mathfrak{h} \cong \mathbb{C}$ for any codimension one Lie subalgebra $\mathfrak{h}$ of $\mathfrak{g}$ gives a point of $B$.

Corollary 3.3. Every line in $\mathbb{P}_{q}(\mathfrak{g})$, not lying in the hyperplane at infinity, intersects $B$.

Proof. Such a line is given by a module of the form

$$
\left(U(\mathfrak{g}) \otimes_{U(\mathfrak{h})} \mathbb{C}_{f}\right)^{\prime},
$$

where $\mathfrak{h}$ is a codimension one Lie subalgebra of $\mathfrak{g}$ and $f \in \mathfrak{h}^{*}$ is a linear form such that $f([\mathfrak{h}, \mathfrak{h}])=0$.

The intersection with the hyperplane at infinity is represented by

$$
\left(U(\mathfrak{g}) \otimes_{U(\mathfrak{h})} \mathbb{C}_{f}\right) \otimes H(\mathfrak{g}) / X_{0} H(\mathfrak{g}) \cong S(\mathfrak{g}) / S(\mathfrak{g}) \mathfrak{h} .
$$

The corresponding point in $\mathbb{P}\left(\mathfrak{g}^{*}\right)$ is given by the quotient map $\mathfrak{g} \rightarrow \mathfrak{g} / \mathfrak{h} \cong \mathbb{C}$. Hence it must be a point of $B$.

Corollary 3.4. If $[\mathfrak{g}, \mathfrak{g}]=\mathfrak{g}$ then the point variety of $\mathbb{P}_{q}(\mathfrak{g})$ consists of

(1) the origin,

(2) the hyperplane at infinity, and

(3) an embedded component at infinity given by $B$.

Proof. Clear from equations (8).

Theorem 3.5. The following are equivalent:

(1) The point variety of $H(\mathfrak{g})$ is reduced, i.e., $Y=Y_{\mathrm{red}}=V\left(\left[x_{i}, x_{j}\right] x_{0} ; 1 \leq\right.$ $i, j \leq n)$.

(2) All lines in $\mathbb{P}_{q}(\mathfrak{g})$ lie on the point variety.

(3) For every codimension one Lie subalgebra $\mathfrak{h}$ of $\mathfrak{g}$ the equality $[\mathfrak{h}, \mathfrak{h}]=$ $[\mathfrak{g}, \mathfrak{g}]$ holds.

Proof. From the results of the foregoing section it is clear that (2) and (3) are equivalent. Let us prove that (1) implies (3). 
First, there can be no codimension one Lie subalgebra $\mathfrak{h}$ such that $[\mathfrak{g}, \mathfrak{g}]$ is not contained in $\mathfrak{h}$, for otherwise there is a point in $A$ not lying on $B$ meaning that there would be an embedded point at infinity. Hence, assume there is a decomposition $\mathfrak{g}=\mathfrak{h} \oplus \mathbb{C} e$ where $\mathfrak{h}$ is a codimension one Lie algebra and [h, $\mathfrak{h}]$ is properly contained in $[\mathfrak{g}, \mathfrak{g}] \subset \mathfrak{h}$. Let $\chi_{\mathfrak{h}}: \mathfrak{g} \rightarrow \mathbb{C}$ be the projection on the second factor. Then, there exists a $\phi \in \mathfrak{g}^{*}$ such that $\phi([\mathfrak{h}, \mathfrak{h}])=0$ whereas $\phi([\mathfrak{g}, \mathfrak{g}]) \neq 0$. But then $\psi=\chi_{\mathfrak{h}}+\varepsilon \cdot \phi$ is a point of $B$ over the dual ring $\mathbb{C}[\varepsilon]$, which is not a point of $A$ over $\mathbb{C}[\varepsilon]$ meaning again that the point variety has an embedded point.

Finally, let us prove that (3) implies (1): we have to show that $A=B$ and as $A$ is smooth it suffices to prove that any point of $B$ over $\mathbb{C}[\varepsilon]$ lies in $A$. So take a surjection $\psi: \mathfrak{g} \rightarrow \mathbb{C}[\varepsilon]$ with the property that

$$
\psi(u) \psi([v, w])+\psi(v) \psi([w, u])+\psi(w) \psi([u, v])=0
$$

Decompose $\psi=\psi_{1}+\varepsilon \psi_{2}$. Then the kernel of $\psi_{1}$ is a codimension one Lie subalgebra $\mathfrak{h}$. Put $\mathfrak{g}=\mathfrak{h} \oplus \mathbb{C} e$ where $\psi(e)=1$. Substitute $u, v \in \mathfrak{h}$ and $w=e$ in the above relation, and as $[\mathfrak{g}, \mathfrak{g}]=[\mathfrak{h}, \mathfrak{h}] \subset \mathfrak{h}$ we obtain $\psi([u, v])=0$, i.e., $\psi([\mathfrak{h}, \mathfrak{h}])=\psi([\mathfrak{g}, \mathfrak{g}])=0$, so it belongs to $A$.

Remark 3.6. With Theorem 3.5 in mind, we pose the question of whether $d$ dimensional linear subspaces (for $d>1$ ) of $\mathbb{P}_{q}(\mathfrak{g})$ arise in a similar way from embedded points in varieties describing lower-dimensional subspaces.

Remark 3.7. Although Theorem 3.5(3) is relatively succinct, it is somewhat unclear to us for which Lie algebras it holds. As the referee pointed out to us, for $\mathfrak{g}$ reductive, it is easy to see that Theorem $3.5(3)$ is true if and only if $\mathfrak{g}$ has no ideal isomorphic to $\mathfrak{s l}(2, \mathbb{C})$.

The case $\mathfrak{g}$ solvable is less clear. Even the case where $\mathfrak{g}$ has a lower central series of length 2 , i.e., $[\mathfrak{g},[\mathfrak{g}, \mathfrak{g}]]=0$, is somewhat technical. Such a Lie algebra is described by the data $(V, W, \phi)$ where $V$ and $W$ are finite-dimensional vector spaces and $\phi$ is a surjective linear map $\wedge^{2} V \rightarrow W . \mathfrak{g}$ is then given by $V \oplus W$ with $\left[(v, w),\left(v^{\prime}, w^{\prime}\right)\right]=\phi\left(v \wedge v^{\prime}\right)$. It is easy to see that any codimension one Lie subalgebra $\mathfrak{h}$ of $\mathfrak{g}$ is of the form $V^{\prime} \oplus W$, where $V^{\prime}$ has codimension one in $V$. Hence $[\mathfrak{h}, \mathfrak{h}]=\phi\left(\bigwedge^{2} V^{\prime}\right)$ whereas $[\mathfrak{g}, \mathfrak{g}]=W$.

Hence Theorem 3.5(3) holds if for any $V^{\prime} \subset V$ of codimension one, $\phi\left(\bigwedge^{2} V^{\prime}\right)$ $=W$. If

$$
\operatorname{dim} W>\operatorname{dim} \bigwedge^{2} V^{\prime}=\frac{(\operatorname{dim} V-1)(\operatorname{dim} V-2)}{2}
$$

this will never be the case and hence there will be an embedded component. On the other hand if $\operatorname{dim} W=1$ and $\phi$ has maximal rank (as a contravariant anti-symmetric tensor) then it is easy to see that there will be no embedded component if $\operatorname{dim} V \geq 4$.

\section{REFERENCES}

1. M. Artin and W. Schelter, Graded algebras of global dimension 3, Adv. Math. 66 (1987), 171-216.

2. M. Artin, J. Tate, and M. Van den Bergh, Some algebras associated to automorphisms of elliptic curves, Grothendieck Festschrift, vol. 1, Birkhäuser, Basel, 1990, pp. 33-85.

3. M. Artin, Geometry of quantum planes, preprint, MIT, 1990. 
4. M. Ascensio, M. Van den Bergh, and F. Van Oystaeyen, A new algebraic approach to microlocalization, Trans. Amer. Math. Soc. 316 (1989), 537-553.

5. J. Dixmier, Enveloping algebras, North-Holland, Amsterdam, 1977.

6. V. Ginsburg, Characteristic varieties and vanishing cycles, Invent. Math. 84 (1986), 327402.

7. L. Le Bruyn and S. P. Smith, Homogenized sl (2), Proc. Amer. Math. Soc. (to appear).

8. Li Huishi and F. Van Oystaeyen, Zariskian filtrations, monograph (to appear).

9. Th. Levasseur, Some properties of non-commutative regular graded rings, Proc. Edinburgh Math. Soc. (to appear).

Department Wiskunde en INFormatica, Universitaire INSTelling ANTWerpen, B-2610 WILRIJK, BELGIUM

E-mail address, L. Le Bruyn: lebruyn@wins.uia.ac.be

Institut des Hautes Etudes SCIEntifiques, 91440 BuRes-SUR-YvetTe, FranCe

Current address, M. Van den Bergh: UFR Mathématique, Université Strasbourg, 7, Rue René Descartes, F-67084 Strasbourg, France

E-mail address, M. Van den Bergh: vdbergh@math.u-strasbg.fr 